\title{
Dinâmica da família no contexto dos cuidados a adultos na quarta idade
}

\author{
Family dynamics in the caring context of adults on the fourth age
}

Dinámica de la familia en el contexto de los cuidados de adultos en la cuarta edad

\section{Sandra Helena Isse Polaro', Lucia Hisako Takase Gonçalves', Silvia Modesto Nassar", Márcia Maria Bragança Lopes', Viviane Ferraz Ferreira'"', Hellen Karinna Monteiro'}

' Universidade Federal do Pará, Faculdade de Enfermagem, Programa de Pós-Graduação em Enfermagem. Belém-PA, Brasil.

"Universidade Federal de Santa Catarina, Centro Tecnológico, Laboratório de Estatística Aplicada. Florianópolis-SC, Brasil.

"II Universidade Federal do Pará, Faculdade de Enfermagem, Programa de Pós-Graduação em Enfermagem (Mestranda). Belém-PA, Brasil. Belém-PA, Brasil.

Submissão: 07-12-2012 Aprovação: 26-03-2013

\section{RESUMO}

Objetivou-se identificar o padrão de funcionamento familiar nas relações de cuidado cotidiano de adultos na quarta idade. Tratase de estudo de natureza diagnóstico-avaliativa de idosos de 80 e mais anos, dependentes de cuidados, e de seus familiares cuidadores, selecionados entre os cadastrados nas unidades de Saúde da Família de um distrito de periferia de Belém-PA, avaliando-os quanto à dinâmica de família, qualidade de vida e estilo de vida relacionado à saúde. Observou-se que a maioria dos idosos avaliou sua família como tendo boa funcionalidade. Porém, os demais dados relativos à qualidade de vida de idosos e cuidadores, como também, estilo de vida dos cuidadores recaíram no nível mediano, levando a inferir certa dificuldade no padrão de funcionamento familiar. Conclui-se que os múltiplos resultados obtidos sinalizam implicações práticas de atenção à unidade familiar e confirmam a necessidade de avaliação multidimensional em intervenção de família.

Descritores: Idoso de 80 e Mais Anos; Família; Enfermagem de Família; Programa Saúde da Família.

\begin{abstract}
This study aimed to determine the pattern of family functioning on everyday care relationships of adults in the fourth age. This is a study of diagnostic-evaluative nature of adults with 80 or more years old who depend on care, and of their relatives as caregivers. The participants were selected among the registered patients of a Family Health Unit in a district in the suburbs of Belém-PA, Brazil. They were evaluated according to the dynamics of their family, and quality of life related health lifestyle. Most of the elderly rated their families with good functionality. However, data on the elderly and caregivers' quality of life and caregivers' life style only reached the median level, showing some difficulty in the family functioning system. It was concluded that the multiple results obtained through the assessments indicate some practical implications of care to the family unity and confirm the need for multidimensional assessment about the family intervention.
\end{abstract}

Key words: Elderly With 80 or More Years; Family; Family Nursing; Family Health Program.

\section{RESUMEN}

Este estudio tuvo como objetivo identificar el patrón de funcionamiento de la familia en las relaciones de cuidado diario de adultos en la cuarta edad. Trata-se de estudio de naturaleza diagnóstico-evaluativo de personas de 80 años o más, dependientes de cuidados, y de sus familiares cuidadores, seleccionados entre aquellos registrados en las unidades de Salud de la Familia, de un distrito de la periferia de Belém-PA. Los mismos fueron evaluados en relación a la dinámica de la familia, calidad de vida y estilo de vida relacionado con la salud. Fue observado que los ancianos, en su mayoría, evaluaron su familia como teniendo una buena funcionalidad. Sin embargo, los demás datos relativos a la calidad de vida de los ancianos y cuidadores, así como al estilo de vida de los cuidadores, fueron de nivel medio, mostrando una cierta dificultad en el padrón de funcionamiento familiar. En conclusión, los múltiples resultados obtenidos en la evaluación señalan implicaciones prácticas para la atención a la unidad familiar y confirman la necesidad de una evaluación multidimensional en la intervención de esta clientela.

Palabras clave: Ancianos de 80 Años o Más; Familia; Enfermería de Familia; Programa de Salud para la Familia.

\section{AUTOR CORRESPONDENTE Sandra Helena Isse Polaro E-mail: shpolaro@ufpa.br}




\section{INTRODUÇÃO}

Em todo o mundo, inclusive nos países em desenvolvimento, as questões oriundas do fenômeno do envelhecimento da população têm levantado várias discussões, notadamente no que concerne às políticas públicas. A transição demográfica no Brasil vem se manifestando com o crescimento acelerado da população idosa, alcançando a taxa de $12,3 \%$ da população geral em 2010 ${ }^{(1)}$ devendo aproximar-se de $30 \%$ em 2050 segundo projeções estatísticas ${ }^{(2)}$.

Evidencia-se o fenômeno do prolongamento da vida no contingente de idosos, ultrapassando os 80 anos de idade nas últimas décadas ${ }^{(3)}$. Enquanto a taxa média geométrica de crescimento anual da população idosa (60 e mais anos) gira em torno de 3,3\%, no estrato de idosos mais idosos (80 e mais anos) essa taxa chega aos 5,4\%, uma das mais altas do mundo(4). A conquista da crescente longevidade humana é um fato positivo do desenvolvimento da sociedade moderna, mas a OMS adverte não ser suficiente acrescentar anos à vida, devendo-se acrescentar mais vida aos anos conquistados. Emerge aí uma demanda quando se observa que o sub estrato de idosos mais idosos, os chamados da quarta idade, tendem a fragilizar-se, integrando a estimativa de $85 \%$ daqueles que sofrem no mínimo de uma doença crônica, dos quais pelo menos $10 \%$ em condições de comorbidade ${ }^{(4-5)}$.

Tal condição vem acompanhada de uma transição epidemiológica singular, de tripla carga de doenças representada por agenda não superada de doenças infecciosas e carenciais, alta carga de causas externas e predomínio de condições crônicas ${ }^{(5)}$. Embora as políticas públicas indiquem ações e programas operativos nesse sentido, o complexo panorama atual e suas consequentes demandas não têm sido contemplados adequadamente a favor das necessidades da população, incluindo a emergente de idosos mais idosos, geralmente devido ao descompasso entre o volume crescente de demanda social e a morosidade com que se operam as mudanças na cultura institucional dos setores da saúde e correlatos para atendê-la.

A prevalência de condições crônicas na população idosa sem o devido acompanhamento e controle contribui para aumentar o número de idosos com limitações funcionais a exigir cuidados prolongados e permanentes, os quais não têm recebido o trato adequado por parte dos serviços de saúde, sobrecarregando a família, tenha ela ou não conhecimentos e habilidades para cuidar do parente idoso ${ }^{(4-5)}$. A tendência das políticas de atenção ao idoso é defender o domicílio como o melhor local para ele viver o seu envelhecimento, a favor da manutenção da autonomia/independência, identidade e dignidade. Realmente, o lar é o espaço sociocultural natural idealizado onde as pessoas geralmente almejam viver. Contudo, a falta de apoio dos serviços sociais e de saúde para o autocuidado da família como unidade, principalmente com membros dependentes de cuidado prolongado, como no caso de idosos fragilizados ou em condições crônicas, torna-se um ônus que ameaça o bem-estar e a qualidade de vida, culminando em desequilibrar a saúde (e as finanças) de toda uma família.

A literatura, internacional e nacional, sobre familiares cuidadores de seus membros dependentes é enfática em demonstrar as consequências, quase sempre danosas, sobre a vida e a saúde dos cuidadores, geralmente mulheres, no desempenho de seu papel. Elas realizam atividades para suprir as demandas de acordo com as necessidades da pessoa dependente. Quaisquer que sejam as circunstâncias de cuidado em contexto familiar, é essencial, além do treinamento específico para o cuidar de outrem, o apoio social dos cuidadores para cuidar de si mesmos. Sem tal suporte, os cuidadores ficam expostos ao risco de adoecer, não pelo cuidado em si, mas pela sobrecarga a que são submetidos ${ }^{(6-9)}$.

Embora a literatura sinalize as múltiplas características da família cuidadora de idosos, o propósito do projeto multicêntrico “DIFAI - Dinâmica da família de idosos mais idosos: o convívio e cuidados na quarta idade em diferentes contextos socioculturais" foi explorar mais a fundo o funcionamento familiar no cuidado de idosos mais idosos, vivendo em diversos contextos.

Em investigações de dinâmica de família, é importante estudar o funcionamento familiar explorando as relações entre seus membros, nas quais se pode observar a relação de harmonia ou não, no funcionamento da unidade de cuidados $^{(9-11)}$.

Entre alguns instrumentos disponíveis, o $\mathrm{APGAR}^{(12-13)}$ de família, eficiente teste de screening de funcionamento familiar, avalia, por meio de cinco questões, as dimensões: Adaptação (adaptation), Companheirismo (partnership), Desenvolvimento (growth), Afetividade (affection) e Capacidade resolutiva (resolve). $\mathrm{O}$ funcionamento familiar refere-se aqui à maneira como a família é vista por seus membros no atendimento desse compromisso (dimensões) e permite identificar as percepções individuais dos valores da família, como recurso psicossocial ou como suporte social. A família saudável é a que demonstra a integralidade desses componentes e representa unidade na sustentação de cuidados ${ }^{(12-13)}$.

As percepções pessoais de cada membro acerca de certos construtos, a exemplo de qualidade de vida e estilo de vida, podem influir na dinâmica da família, fazendo-a mais ou menos adaptativa, mais ou menos funcional, diante de uma situação que se apresenta como fato novo a enfrentar, criando impactos sobre as relações intrafamiliares. Por isso, optou-se neste estudo, avaliar também a qualidade de vida, na concepção da $\mathrm{OMS}^{(14-15)}$, de ambos: idoso e familiar cuidador, e o estilo de vida relacionado à saúde - uma concepção desenhada por Nahas ${ }^{(16)}$ - do familiar cuidador, presumindo-se que, quanto mais saudável for o estilo de vida, melhores serão as relações de cuidado com o parente idoso.

A qualidade de vida ${ }^{(14-15)}$ representa a percepção individual sobre a posição na vida no contexto da cultura e do sistema de valores nos quais o indivíduo se insere e em relação a seus objetivos e expectativas, padrões e preocupações. Esse fator tem valor subjetivo importante, pois influi diretamente em sua condição de bem-estar e de saúde, assim como na sensação de maior ou menor competência para administrar a própria vida e quaisquer outras circunstâncias que cercam o idosos ou nas quais se encontra, como na família. Já o estilo de vida de um indivíduo corresponde a um conjunto de ações habituais que refletem atitudes, valores e oportunidades. Essas ações, que compreendem comportamentos nutricionais, de atividades físicas e controle de estresses, controle de saúde, 
de relacionamentos sociais, têm grande influência na saúde geral e, por sua vez, na qualidade de vida ${ }^{(16)}$.

\section{METODOLOGIA}

Trata o presente artigo de parte de um projeto maior, o DIFAI, mencionado anteriormente, realizado no contexto de Belém/PA, com o objetivo de identificar padrão de funcionamento familiar nas relações de cuidado cotidiano de idosos da quarta idade.

O contexto do estudo, Belém, capital do estado do Pará, possui população de 1.393.399 habitantes, com estrato idoso representando 9,3\% do total, taxa superior à média do Estado. Quanto aos idosos de 80 e mais anos já atingem 18.273 pessoas em números absolutos e representam $16,3 \%$ da população idosa $^{(1)}$.

A abordagem do estudo, uma pesquisa descritiva de natureza diagnóstico-avaliativa, foi realizada com idosos de 80 e mais anos, dependentes de cuidados de outrem e, com seu respectivo familiar cuidador principal. A definição de tal cuidador se deu pela auto denominação.

A amostra foi obtida por conveniência, localizando-se os sujeitos pares: idoso e seu familiar cuidador principal, em cinco unidades de Estratégia de Saúde da Família (ESF), sediadas num distrito periférico empobrecido de Belém, onde há grande concentração de idosos cadastrados.

A coleta de dados se deu entre 08/2009 e 07/2010, de uma amostra de 110 pares (idoso de 80 e mais anos e familiar cuidador principal) que, tendo condições de responder às perguntas, foram convidados e aceitaram participar do estudo.

O QPFC - Questionário de Perfil da Família Cuidadora ${ }^{(6)}$ foi aplicado ao familiar cuidador para as questões de identificação: variáveis sociodemográficas, situações de saúde e cuidados, de ambos (idoso e cuidador).

Para conhecer o padrão de funcionamento da família nas relações de cuidado, delimitou-se avaliar a dinâmica da família, na óptica dos idosos, aplicando-se a escala APGAR ${ }^{(12-13)}$; e sua qualidade de vida, aplicando-se o questionário WHOQOL ${ }^{(14-15)}$. Já na óptica dos familiares cuidadores, foram avaliados seu estilo de vida, aplicando-se a Escala de Nahas ${ }^{(16)}$ e, também, sua qualidade de vida ${ }^{(14-15)}$.

A escala APGAR de família ${ }^{(12-13)}$, de fácil aplicação, comporta cinco questões, com opções de resposta: sempre, quase sempre, algumas vezes, raramente e nunca, com pontuação de respectivamente quatro, três, dois e um ponto e zero. A avaliação pelo valor da pontuação total obtida (amplitude de escores de 0 a 20), classifica a família em três tipos: altamente funcional (escores 13 a 20), moderadamente funcional (escores 9 a 12) e com disfunção acentuada (escores 0 a 8). A validade e confiabilidade dessa escala garantem segurança em sua aplicação ${ }^{(13)}$.

A avaliação da qualidade de vida se deu aplicando-se ao familiar o WHOQOL-Breve e ao idoso o WHOQOL-Breve mais o WHOQOL-Old, instrumentos da OMS para avaliação da qualidade de vida relacionada à saúde ${ }^{(14-15)}$. A versão WHOQOL-Breve, de 26 quesitos, abrange quatro domínios de qualidade de vida e preserva as vinte e quatro facetas do original WHOQOL-100.
Cada questão tem cinco opções de resposta do tipo Likert, oscilando de 1 a 5 pontos. O WHOQOL-Old, de 24 quesitos, inclui facetas específicas e sua avaliação se faz de modo análogo à anterior. Todas as variantes do WHOQOL têm bons índices de validade e confiabilidade ${ }^{(14-15)}$. Seus escores são avaliados pela posição tomada na amplitude entre zero e 100, considerando quanto maior a pontuação, melhor a qualidade de vida.

Ademais, para os familiares aplicou-se a Escala de Nahas ${ }^{(16)}$, que avalia o estilo de vida de comportamentos que afetam a saúde, com 15 questões compreendendo cinco ações habituais: Nutrição, Atividades físicas, Comportamentos preventivos para a saúde, Relações sociais e Controle de estresse, que refletem atitudes, valores e oportunidades das pessoas. A escala possui validade e confiabilidade aceitáveis ${ }^{(16)}$. O escore obtido é avaliado pela posição tomada na amplitude entre zero e 45 , correspondendo respectivamente a inadequado estilo de vida e ótimo estilo de vida.

O projeto foi submetido ao Comitê de Ética em Pesquisa com Seres Humanos da UFSC - Universidade Federal de Santa Catarina - (sede da coordenação do projeto DIFAI), seguindo as recomendações da Resolução 196/96 do Conselho Nacional de Saúde do Ministério da Saúde e aprovado por parecer exarado no processo protocolado sob n. 051/08.

\section{RESULTADOS}

Caracterização da Amostra - A amostra, composta por 110 idosos de 80 anos e mais, e por 110 familiares cuidadores, encontra-se caracterizada na Tabela 1.

A maioria dos idosos é composta de mulheres, predominantemente viúvas, porém há ainda 23,6\% (26) de casados dos quais 14 são cuidadores de seus próprios cônjuges com média de idade de 82 anos.

Tabela 1 - Caracterização sociodemográfica, de saúde, e de avaliação de qualidade de vida, APGAR de família e estilo de vida dimensionados em escores obtidos por idosos mais idosos (80 e mais anos) e por familiares cuidadores, Belém/PA, 2010.

\begin{tabular}{lcc}
\hline Variáveis & $\mathbf{n}(\mathbf{1 1 0})$ & \% \\
\hline \multicolumn{1}{l}{ Idoso de $\mathbf{8 0}$ anos e mais $(\mathbf{n}=\mathbf{1 1 0})$} & & \\
Idade em estrato & 57 & 51,8 \\
$\quad 80-84$ anos & 26 & 23,6 \\
$85-89$ anos & 27 & 24,6 \\
$\quad 90$ anos e + & 31 & 28,2 \\
Sexo $\quad$ Masculino & 79 & 71,8 \\
$\quad$ Feminino & & \\
Escolaridade & 35 & 31,8 \\
$\quad$ Analfabeto (a) & 55 & 50,0 \\
$\quad$ Ensino primário até 4 anos & 17 & 15,4 \\
$\quad$ Ensino ginasial até 8 anos & 3 & 2,8 \\
$\quad$ Ensino superior: incompleto/completo &
\end{tabular}


Estado Conjugal

Casado(a)

26

Viúvo(a)

Divorciado(a)/solteiro(a)

Idade do cônjuge $(\mathrm{n}=26)$ média/DP

Parentesco do familiar cuidador

Filha(o)

$\operatorname{Neta}(0)$

Cônjuge

Doenças de que sofre

Hipertensão arterial
Sequela de AVC
Cardiopatia
Diabetes mellitus
Osteoporose
Outras

Apgar de Família (Amplitude dos escores 0-20)

Boa funcionalidade (escores de 13-20)

Moderada funcionalidade (escores de 9-12)

Acentuada disfuncionalidade (escores de 0-8)

Qualidade de vida - WHOQOL-Breve (0-100) Escore percentual médio/DP

$56,3 / 14,8$

$61,3 / 14,3$

Qualidade de Vida - WHOQOL-Old (0-100)

Escore percentual médio/DP

Familiar cuidador do idoso $(n=110)$

Idade (média em anos/DP)

$47 / 16,5$

Sexo

Masculino

Feminino

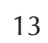

97

11,8

88,2

Estado Conjugal

Casado(a)

Solteiro(a)

Viúvo(a)/Divorciado(a)

Ocupação/Trabalho: tem

Cuida de outros dependentes - sim

Mora com o idoso cuidado - sim

Qualidade de Vida-WHOQOL-Breve ( 0-100)

Escore percentual médio / DP

$43 \quad 39,1$

$13 \quad 11,8$

$27 \quad 24,5$

$68 \quad 61,8$

$79 \quad 71,8$

$63,3 / 11,0$

Estilo de vida (0-45)

Escore percentual médio/DP

$23,5 / 8,2$

A amostra de familiares cuidadores revelou-se predominantemente feminina, com média de idade em torno de 47 anos e representada principalmente por filhas e netas. Além de cuidar do idoso, a maioria tem também sob seu encargo o cuidado de outras pessoas dependentes na família $(61,8 \%)$. Há ainda quem trabalhe fora de casa, em 24,7\%. Observa-se que a maioria $(71,8)$ vive na mesma casa do idoso que cuida.

Na Tabela 2 apresentam-se os escores médios de qualidade de vida obtidos nos domínios correspondentes, dimensionando as variações das percepções entre idoso e cuidador.

Tabela 2 - Média e desvio padrão dos escores percentuais nos domínios do WHOQOL-Breve (amplitude 0-100) obtidos pelos idosos e familiares cuidadores, do contexto de Belém/ PA, 2010.

\begin{tabular}{lcccc}
\hline \multirow{2}{*}{$\begin{array}{c}\text { WHOQOL - Breve } \\
\text { Domínios }\end{array}$} & \multicolumn{2}{c}{ Média } & \multicolumn{2}{c}{ Desvio padrão } \\
\cline { 2 - 5 } & Idoso & Cuidador & Idoso & Cuidador \\
\hline Físico & 54,0 & 70,9 & 19,9 & 15,5 \\
Psicológico & 61,4 & 67,1 & 17,8 & 12,8 \\
Relações Sociais & 62,7 & 68,4 & 16,0 & 14,9 \\
Meio Ambiente & 50,3 & 52,2 & 15,5 & 15,0 \\
\hline
\end{tabular}

A resposta dos idosos ao WHOQOL-Breve (escore total médio 53,4$)$ mostrou pontuação mais baixa nos domínios Físico e Meio Ambiente, enquanto os cuidadores (escore total médio $62,4)$ se avaliaram com pontuação mais baixa nos domínios Psicológico e Meio Ambiente. Esses resultados indicam possíveis deficiências no contexto domiciliar, dificuldades no convívio relacional de cuidados, cada qual percebendo-se mais afetados nos domínios apontados.

\section{DISCUSSÃO}

A predominância de idosas sobre idosos na amostra confirma a tendência da já conhecida feminização da velhice ${ }^{(1-3)}$. A baixa escolaridade encontrada já era previsível, considerando a realidade pregressa de quem hoje conta 80 ou mais anos de idade. A constatada prevalência de afecções crônicas se constitui em panorama epidemiológico comum à população envelhecente $^{(5)}$. O fato irremediável de conviver permanentemente com as condições crônicas pelo aumento da longevidade pode fazer os idosos perderem qualidade de vida, pois as atuais políticas públicas de rede de cuidados continuados e integrados ${ }^{(4-6)}$ para atenção à vida e à saúde dos idosos estão ainda longe de serem efetivos. É importante que se dê cobertura prioritária às famílias mais empobrecidas, que dependem do único recurso da aposentadoria/pensão do idoso, pois se observou que $71,8 \%$ dos cuidadores viviam na mesma casa que os idosos, principalmente pelo empobrecimento das famílias.

Aqui os idosos são ainda majoritariamente cuidados por mulheres $(71,8 \%)$, como já constatado em estudos anteriores e também na replicação do presente estudo em outros contextos brasileiros ${ }^{(6,17-18)}$. Já em Portugal, na cidade do Porto, revelou-se o despontar do cuidador masculino em $40 \%$, entre fiIhos e sobrinhos jovens, e até maridos idosos ${ }^{(17)}$. Raros estudos de cuidadores masculinos, inclusive de idosos cuidadores de suas esposas doentes ${ }^{(17,19)}$, revelaram especial dedicação, contrariando o tradicional estereótipo de gênero que atribui o cuidado da família basicamente à mulher. É possível, e é preciso 
encorajar homens à tarefa familiar, de modo a contribuir para reduzir o estresse de cuidadoras únicas na família( ${ }^{(17-18)}$. Os dados com destaque especial de cônjuges cuidadores de ambos os sexos $(12,7 \%)$ aqui encontrados corroboram resultados de alguns estudos ${ }^{(6-7,11,17-18)}$ que demonstram tendência a aumentar com o escasseamento de familiares descendentes.

Os familiares cuidadores são muitas vezes tomados por sentimentos de solidão e isolamento social, como sinalizaram aqui os escores mais baixos na avaliação da qualidade de vida nos domínios psicológico e meio ambiente. Por isso, na prática, é necessário articular redes locais de suporte social, a partir dos serviços de saúde, para ajudar a manter a inclusão social das famílias cuidadoras. Perguntados sobre como percebem sua própria saúde, os familiares cuidadores responderam ser boa em $57,2 \%$, mas para $42,8 \%$ é regular ou má, correspondendo à avaliação geral da qualidade de vida e do estilo de vida, com seus escores em nível mediano, possivelmente devido ao estresse e sobrecarga restando-lhes pouco tempo para o cuidado de si, e daqueles já idosos, acrescido do desgaste do próprio envelhecimento, o que é confirmado em pesquisas estrangeiras e nacionais s $^{(6-9,17-20)}$.

A dinâmica de família avaliada pelo APGAR neste estudo revelou que a maioria dos idosos (85\%) vê sua família com boas relações e boa funcionalidade. Esse dado, porém, pode ser lábil, cabendo alertar para a eventual desestabilização da dinâmica de relações familiares. Por isso, sempre é lugar para intervenção antecipativa de prevenção de possíveis crises na família ${ }^{(13,20)}$. Assim, cabe intervenção de família pelos profissionais, sobretudo enfermeiros, que estão envolvidos mais diretamente na gestão do cuidado e na administração de dificuldades familiares. Quando o idoso relata uma crise familiar, geralmente os recursos familiares estão inadequados para suprir as necessidades ou existem áreas vulneráveis que podem interferir na habilidade das famílias em encontrar estratégias e recursos para cumprir seu papel de cuidador ${ }^{(11,19-20)}$. De outra parte, as percepções pessoais de cada membro da família acerca de certos construtos como qualidade de vida e estilo de vida, influem na dinâmica da família, fazendo-a mais ou menos adaptativa, mais ou menos funcional, diante de uma situação que se apresenta como fato novo a enfrentar: um membro idoso que adoece e se torna cada dia mais frágil e dependente de cuidados, criando impactos sobre as relações intrafamiliares.

O escore médio de estilo de vida relacionado à saúde obtido pelos familiares cuidadores situou-se em nível mediano, assim como o resultado regular de qualidade de vida, denotam riscos ao bom funcionamento familiar e requer aqui o cuidado da família como uma unidade ${ }^{(11,20)}$.

O dimensionamento dos resultados pelo WHOQOL-Breve de idosos e cuidadores (Tabela2) bem demonstra os domínios de qualidade de vida mais afetados: Meio Ambiente, Físico e Psicológico, o que é corroborado por resultados de outros estudos $^{(7,9,11,19-20)}$. Ambientes domésticos de famílias empobrecidas não favorecem condições mínimas para cuidados básicos diários, condicionando desconforto para os idosos e, consequentemente, aborrecimento por parte dos familiares cuidadores no cumprimento de suas tarefas.

As duas versões do WHOQOL, Breve e Old, foram aqui aplicadas porque os autores validadores do questionário ${ }^{(14-15)}$ recomendam aplicá-las em conjunto para idosos, com a vantagem de a última avaliar as especificidades das percepções mais positivas ou negativas nas facetas dos domínios de qualidade de vida. A análise da associação entre os escores obtidos no WHOQOL-Breve $(55,7)$ e WHOQOL-Old $(61,43)$, testada pelo coeficiente de correlação de Spearman, devido a não distribuição normal, resultou em rs 0,8105 ( $p$ valor $=0.00445$ ) com significância estatística $p<0,000001$, evidenciando relação positiva entre os escores de ambos os questionários, ou a equivalência dos resultados. Mesmo resultado foi encontrado na análise dessa associação nos diferentes contextos repli$\operatorname{cados}^{(17-18,20)}$, o que reforça a equivalência das duas versões do WHOQOL. Tal constatação permite recomendar com mais segurança a aplicação independente de uma das versões do WHOQOL, especialmente para aqueles idosos que se cansam em responder as 50 questões em aplicação conjunta.

\section{CONCLUSÃO E IMPLICAÇÕES PRÁTICAS}

O padrão de convívio familiar entre o idoso e seu cuidador, avaliado sob a óptica de ambos, leva-nos a inferir que, embora a maioria dos idosos refira ser de boa funcionalidade a dinâmica de sua família, as respostas de nível mediano, tanto de estilo de vida dos cuidadores quanto as de qualidade de vida de ambos, refletem possíveis dificuldades ou riscos ao convívio em face das relações de cuidado.

Os resultados obtidos em amostra por conveniência impedem sua generalização. Porém, os resultados semelhantes obtidos dessa pesquisa quando replicada em diferentes contextos socioculturais trazem contribuições de ordem prática, principalmente para a enfermagem de família priorizar: a) necessidade de acolher a família cuidadora não como simples parceira de cuidados, mas como unidade a ser cuidada; b) atenção especial da saúde das(os) cuidadoras(es), prevenindo adoecimentos; c) aconselhamento de rodízio de cuidadores entre membros da família - sem esquecer os homens, com orientações específicas na preservação da qualidade de vida possível entre seus membros, e manutenção de estilo de vida saudável; d) avaliação multidimensional, imprescindível para a atenção integral da unidade familiar; e) suporte ao autocuidado da família, valendo-se de recursos tecnológicos cuidativo-educativos possíveis na assistência da família.

\section{REFERÊNCIAS}

1. Instituto Brasileiro de Geografia e Estatística [homepage na internet]. Sistema IBGE de Recuperação Automática (SIDRA). Censo Demográfico 2010 - Tabela 3107:
População residente, por situação do domicílio, sexo e grupos de idade - Sinopse [acesso em 4 mar 2011]. Disponível em: www.sidra.ibge.gov.br 
2. Instituto Brasileiro de Geografia e Estatística [homepage na internet]. Projeção da população do Brasil por sexo e idade, 1980-2050: revisão 2008 [acesso em 04 mar 2011]. Disponível: http://www.ibge.gov.br/home/estatistica/populacao/projecao_da_populacao/2008/default.shtm.

3. Camarano AA, organizadora. Os novos idosos brasileiros: muito alem dos 60?. Rio de Janeiro: IPEA; 2004.

4. Camarano AA, editor. Cuidados de longa duração para a população idosa: um novo risco social a ser assumido?. Rio de Janeiro: IPEA; 2010.

5. Mendes, E V. O cuidado das condições crônicas na atenção primária à saúde: o imperativo da consolidação da estratégia da saúde da família. Brasília: Organização Pan-Americana de Saúde; 2012.

6. Gonçalves LHT, Portella MR, Mendes AC, et al. Profile of the family caregiver for a frail elderly relative in the domestic environment at the five sociocultural contexts of Florianopolis/SC, Jequié/BA, Passo Fundo/RS, Rosário/Ar and Porto/Pt. In: Program of the $18^{\text {th }}$ Congress of the International Association of Gerontology[ evento na internet]. 2005 jun 28-30; Rio de Janeiro, Brasil [CD-ROM]. Rio de Janeiro: SBGG, 2005.

7. Rosset I, Pedrazzi EC, Roriz-Cruz M, Morais EP, Rodrigues RAP. Tendências dos estudos com idosos mais velhos na comunidade: uma revisão sistemática (inter)nacional. Rev Esc Enferm USP 2011;45(1):264-71.

8. Feliciano AB, Moraes SA, Freitas ICM. O perfil do idoso de baixa renda no município de São Carlos, Brasil: um estudo epidemiológico. Cad Saúde Pública 2004;20(6):1575-85.

9. Oliveira DC, D'Elboux MJ. Estudos nacionais sobre cuidadores familiares de idosos: revisão integrativa. Rev Bras Enferm 2012;65(5):829-38.

10. Souza AS, Meira EC, Neri IG, Silva JA, Gonçalves LHT. Fatores de risco de maus tratos ao idoso na relação idoso/ cuidador em convivência intrafamiliar. Textos Envelhecimento $2004 ; 7(2): 63-85$.
11. Silveira CL, Budó MLD, Silva FM, Beuter M, Schimit MD. Rede Social das cuidadoras de familiares com doença crônica incapacitante no domicílio: implicações para a enfermagem. Cienc Cuid Saúde 2009;8 (4):667-74.

12. Gardner W, Nutting PA, Kelleher KJ, Werner JJ, Farley T, Stewart $L$, et al. Does the family APGAR effectively measure family functioning?. J Fam Pract 2001;50(1):19-25.

13. Duarte YAO. Família: rede de suporte ou fator estressor: a ótica de idosos e cuidadores familiares. Tese [Doutorado em Enfermagem] -Escola de Enfermagem de São Paulo da Universidade de São Paulo; 2001.

14. Fleck MP, Louzada S, Xavier M, Chachamovich E, Vieira G, Santos L, Pinzon V. Application of the Portuguese version of the abbreviated instrument of quality life WHOQOL-bref. Rev Saúde Pública 2000;34(2):178-83.

15. Fleck MPA, Chachamovich E, Trentini CM. Projeto WHOQOL-OLD: método e resultados de grupos focais no Brasil. Rev Saude Publica 2003;34(6):793-9.

16. Nahas MV, Barrros MVG, Francalacci V. Pentáculo do bem estar: base conceitual para avaliação do estilo de vida de indivíduos e grupos. Rev Bras Ativ Fis Saúde 2000;5(2):48-59.

17. Gonçalves LHT, Costa MAM, Martins MM, Nassar SM, Zunino R. The family dynamics of elder elderly in the context of the Porto, Portugal. Rev Latino-Am Enferm 2011;19(3):458-66.

18. Gonçalves LHT, Nassar SM, Daussy MFS, Santos SMA, Alvarez AM. O convívio familiar do idoso na quarta idade e seu cuidador. Cienc Cuid Saúde 2011;10(4):746-754.

19. Chaves L, Pedreira A, Oliveira M. Cuidadores de idosos dependentes no domicílio: mudanças nas relações familiares. Rev Bras Enferm 2012;65(5):730-6.

20. Falcade BL, Leite MT, Hildebrandt LM, Kirchner RM, Silva LAA. Gonçalves LHT. Octogenários em residências unipessoais: enfoque sobre a qualidade de vida e condições de saúde. Rev Enferm UFSM 2011;1(3):386-393. 